\title{
Le modèle Bassidji (Partie 1)
}

\author{
Farhad Khosrokhavar
}

\section{(2) OpenEdition}

1 Journals

\section{Édition électronique}

URL : http://journals.openedition.org/conflits/680

DOI : $10.4000 /$ conflits.680

ISSN : 1777-5345

Éditeur :

CCLS - Centre d'études sur les conflits lilberté et sécurité, L'Harmattan

\section{Édition imprimée}

Date de publication : 15 octobre 1998

ISSN : 1157-996X

\section{Référence électronique}

Farhad Khosrokhavar, «Le modèle Bassidji (Partie 1) », Cultures \& Conflits [En ligne], 29-30 | automnehiver 1998, mis en ligne le 16 mars 2006, consulté le 30 mars 2021. URL : http://

journals.openedition.org/conflits/680; DOI : https://doi.org/10.4000/conflits.680

Ce document a été généré automatiquement le 30 mars 2021.

Creative Commons License 


\title{
Le modèle Bassidji (Partie 1)
}

\author{
Farhad Khosrokhavar
}

Dans la période révolutionnaire, s'échelonnant grosso modo de l'année 1978 à la mort de Khomeyni (juin 1989) se développe, avec une intensité jamais atteinte dans l'histoire iranienne en général et dans celle de l'islam en particulier, le phénomène du martyre. Que ce soit dans la lutte contre le chah ou dans la guerre contre l'Irak qui est l'une des plus longues de la seconde moitié du XXe siècle (1980-1988) et qui laisse un million de morts et de blessés des deux côtés ${ }^{1}$, le martyre est omniprésent, comme phénomène culturel mais aussi politique et social chez les jeunes Iraniens (la plupart sont des " déshérités ", (mostaz'afin), une jeunesse de couches inférieures ou moyennesinférieures, urbanisée depuis une ou deux générations). Cet islam politique où le martyre chiite (chahâdat) occupe une place de choix est d'abord est perçu comme une spécificité sinon iranienne, du moins chiite. La radicalisation chiite au Sud Liban, où certains groupes se lancent dans des attentats suicidaires contre l'armée d'occupation israélienne, corroborent cette idée. Il faudra les années quatre-vingt-dix, le développement du Hamas et du Djihad en Palestine et des courants extrémistes issus de la désintégration du FIS en Algérie (GIA, etc.) pour que se dissipe cette vision du martyre comme étant indissolublement lié au chiisme.

Ce travail entend souligner les causes sociales de l'émergence de cette mort sacrée dans la religiosité des jeunes, et marquer la différence essentielle qui sépare le néo-martyre juvénile du martyre traditionnel ${ }^{2}$.

La révolution en dérive

A partir du début des années quatre-vingt, la société post-révolutionnaire est confrontée à une conjoncture de guerre mais aussi à une situation économique et politique de plus en plus difficile, en contradiction flagrante avec les espérances soulevées à l'aube de la révolution où on promettait à chacun " son baril de pétrole ", son " droit au pétrole " spolié par le régime impérial, ou bien encore un développement harmonieux après la disparition du régime " démoniaque " du chah.

L'état de la société, à peine un an après la révolution, est en contradiction flagrante avec les rêves caressés au début du mouvement révolutionnaire : l'économie, dans la première année relativement indemne, sombre progressivement dans un état de 
léthargie, puis, avec la guerre lancée par l'Irak principalement dans les zones pétrolifères de l'Iran (Khouzistan) et les incertitudes politiques liées à la radicalisation $\mathrm{du}$ mouvement révolutionnaire, régresse visiblement. En même temps, en contradiction avec l'idéal de la justice que promettait la révolution, se trouve jetée la base d'une économie hyper-spéculative où s'enrichissent rapidement des groupes parasites alors que l'inflation et le chômage laminent le niveau de vie des couches moyennes et inférieures.

La nationalisation de grands pans de l'industrie et l'expropriation des industriels " en fuite ", l'incurie post-révolutionnaire et la migration progressive des membres des classes moyennes où se recrutaient le plus clair du personnel technique et scientifique, jettent la société dans une situation où l'incompétence généralisée est compensée, au début des années quatre-vingt, par la surenchère dans le politique, défini lui-même en termes théologiques. Une atmosphère morose doublée du sentiment d'avoir été trahi, pour les uns par la contre-révolution, pour les autres par les tenants du nouveau pouvoir, règne dans une société traumatisée par les échecs successifs du mouvement révolutionnaire: échec social (approfondissement de la pauvreté et surtout, élargissement du fossé entre pauvres et riches, entre les affidés du pouvoir et les laissés pour compte), économique (une économie spéculative transfère les ressources vers des activités non-productives), culturel (la morosité régnante se marque par un puritanisme exacerbé qui discrédite, pour de larges pans de la société, un pouvoir " rabat-joie "). La guerre rend encore plus difficile la situation, exposant les jeunes à la mort et mettant en danger les zones pétrolifères (le Khouzistan, au sud-est de l'Iran) d'où l'Etat tire le plus clair de ses ressources.

A peine deux années après la révolution de 1979, la société se trouve jetée, démunie et sans capacité de mobilisation, dans une guerre meurtrière tandis que le pouvoir se légitime par sa capacité de mobiliser les jeunes dont la radicalisation exprime la riposte chaude du mouvement révolutionnaire à la dégradation des relations socioéconomiques.

C'est sur fond d'une révolution en dérive, d'une guerre meurtrière, d'une situation sociale de plus en plus malaisée et d'une économie en régression que le modèle bassidji trouve son sens. Il exprime le sentiment que l'on est proche de la fin des temps, il traduit le désespoir présent qui, en s'approfondissant, présage d'une fin " heureuse ". Pour une catégorie spécifique de jeunes, la vie n'a plus aucun attrait ni aucun sens, et c'est dans une mort " digne " que le sens se conquiert, plutôt que dans une vie par définition " indigne ". La mort, digne présage d'un futur post-mortem irénique ! et dès lors, la tâche " islamique " par excellence consiste à tout faire pour mourir et préparer les autres, de gré ou de force, à embrasser la mort. Certes, seule une minorité pense de la sorte parmi les jeunes révolutionnaires, mais cette minorité-là n'est pas n'importe laquelle, elle est le fer de lance de la révolution et surtout, manipulée par l'Etat qui y découvre un principe de légitimation mortifère, elle se trouve mise en vedette par tous les moyens dont dispose le pouvoir, à savoir les médias écrits et la télévision qui consacre de longues heures aux " récits du front de guerre ". Propagande et héroïsation font de ces jeunes en quête de la mort les " stars " d'une modernité absente. Quant à l'Etat post-révolutionnaire, il puise dans la glorification des martyrs morts sur le front ou des " martyrs vivants " (chahidé zéndéh, ceux qui ont été gravement blessés ou ceux qui ont survécu à des massacres perpétrés sur le front par l'armée irakienne, par exemple à l'occasion d'un bombardement chimique) une légitimité répressive qu'il utilise contre tous ceux qui voudraient contester son mode d'action ou simplement lui 
rappeler les promesses de la révolution en matière de justice sociale et de liberté - le slogan de la révolution a été " Liberté, indépendance, République islamique ".

Le chiisme et sa prise en charge par la révolution

Le chiisme qui opère comme idéologie mobilisatrice de la révolution iranienne n'est pas la reproduction ne varietur du chiisme traditionnel. En son sein de nombreuses transformations se sont opérées qui en ont fait une arme de combat aux mains de nombreuses couches de la population. Le processus de sécularisation a débuté vers la fin du XIXe siècle pour se radicaliser à partir des années soixante-dix, notamment en la personne de Shariati qui devient l'idéologue chef du mouvement révolutionnaire ${ }^{3}$ et dont les expressions - comme " autoconstruction révolutionnaire " (khodsâzié enghélâbi), le chiisme rouge (tachayyo'é sorkh), chiisme d'Ali (tachayyo'é alavi)...- sont devenues monnaie courante dans la révolution iranienne. La sécularisation du chiisme ${ }^{4}$ renverse le rôle du croyant : naguère, il était passif et dans l'attente quiétiste de l'Imam du temps (le Douzième Imam occulté) selon Shariati, infesté en cela par des pouvoirs qui causaient son aliénation (" l'abêtissaient ", estéhmâr) ; dès lors, le croyant épousait une attitude doloriste en s'exilant mentalement dans une Fin des temps qui laissait aux régimes despotiques et injustes tout le loisir de dominer le peuple ici-bas. A présent, le croyant authentique transforme l'attente passive en attente active, le chiisme des tenants du pouvoir inauthentique des classes dominantes (le chiisme des Séfévides, tachayyo'é safavi), en un chiisme authentiquement révolutionnaire (le chiisme d'Ali, tachayyo'é alavi) et ce, en inaugurant sa lutte révolutionnaire contre les tenants de l'injustice sociale. Pour Shariati, cette attitude qui s'inscrit dans la vocation originaire du chiisme, hâte la venue de l'Imam occulté ${ }^{5}$. Pour la première fois, en ce qui concerne la jeunesse urbaine en voie de modernisation, l'appel se trouve lancé pour qu'elle devienne sujet de l'Histoire plutôt que réceptacle passif et éploré d'un monde injuste. Combiné à la version ambivalente que donne Khomeyni d'un chiisme politique, cet appel est l'un des leit-motifs qui mobilisent les jeunes contre le régime pahlavi. On est face à une politisation du Sacré, qui demeurera, jusqu'au bout, l'une des caractéristiques essentielles des martyrs de la révolution, les " Bassidji " ${ }^{6}$ : après la révolution et le commencement de la guerre avec l'Irak (1980), les ressorts de l'idéologie chiite vont être mis à contribution pour donner une justification au martyre des jeunes sur le front et surtout, pour apporter du lustre à un pouvoir qui, tout au long de la guerre, n'a pu qu'organiser le déclin socio-économique de la société iranienne ${ }^{7}$.

Le martyre, dans le chiisme, joue un rôle autrement important que dans le sunnisme, branche principale de l'islam ${ }^{8}$. L'histoire de la vie des imams chiites est marquée par les martyrs réels ou supposés, la quasi-totalité de ces personnages saints ayant succombé, réellement ou dans l'imaginaire des croyants, aux sévices du pouvoir. Cependant, contrairement à certaines interprétations modernisées du chiisme qui mettent l'accent sur son soi-disant aspect proto-révolutionnaire $\mathrm{du}$ fait de son caractère eschatologique ${ }^{9}$, pendant longtemps, à partir de son instauration en Iran comme religion d'Etat sous les Séfévides, en réalité, le chiisme a eu une dimension quiétiste prédominante: l'attente de l'Imam du Temps (autre surnom du Douzième Imam occulté) discréditait indistinctement tout pouvoir temporel (c'était un pouvoir autre que celui de l'Imam occulté), mais dans le même mouvement, lui laissait les coudées franches, dans la mesure où l'histoire sainte se déroulait sur un autre plan, dans une hiéro-histoire invisible qui avait sa propre légalité. Tout pouvoir étant " illégitime " par essence sur le plan doctrinal, aucun pouvoir n'était, de facto, illégitime, ce qui donnait une grande latitude aux diverses formes de pouvoir qui s'étaient déployées en Iran, et 
qui laissaient ${ }^{10}$ se déployer le dolorisme chiite. Moyennant cet accommodement minimal et la prise en charge financière et politique d'une partie des ulémas (les imams djom'és), le pouvoir se trouvait, en réalité, largement affranchi de l'emprise du religieux. Un modus vivendi s'établissait ainsi entre les autorités religieuses et les régimes politiques qui, sauf en période de trouble ou d'instabilité (rois fainéants, changement de dynasties, compétition pour le pouvoir de plusieurs prétendants...), laissait une grande latitude à l'Etat ${ }^{11}$. Avec l'emprise croissante des puissances étrangères en Iran (la Grande-Bretagne au sud et la Russie tsariste au nord du pays) à partir du début du XIXe siècle, une rénovation progressive prend place au sein de l'idéologie religieuse et à partir de la seconde moitié du XIXe siècle, la fonction contestataire s'actualise; alors que jusque là, elle se cantonnait à la Fin des Temps (l'Imam du temps allait renverser les pouvoirs injustes juste avant le Jugement dernier, le croyant n'avait qu'à attendre). Le clergé et une partie des couches urbaines sécularisent la fonction eschatologique du chiisme et donnent un sens intra-mondain à " l'illégitimité du pouvoir " au sein du chiisme. Celui-ci devient l'un des trois pôles majeurs où se construit le rapport au politique, avec le nationalisme, balbutiant au début du XXe siècle, de plus en plus important à partir des années trente, et le marxisme dont l'influence sur les intellectuels iraniens, en particulier du fait de la proximité de la Russie et ensuite, de l'Union Soviétique, sera extrêmement forte jusqu'à la Révolution Islamique.

La vague islamiste déferle sur la société iranienne après une période d'intense modernisation socio-économique et de fermeture accrue du champ politique d'où sont exclues les nouvelles couches sociales issues de la modernisation. Le mouvement d'opposition au chah a perdu, à partir du début des années soixante-dix, son caractère démocratique, du moins dans ses composantes essentielles, du fait même de la répression dont les tenants du pluralisme politique ont été les victimes, aux côtés des gauchistes et des islamistes ${ }^{12}$. Par ailleurs, l'opposition islamique s'est radicalisée après la répression des années soixante et notamment celle du mouvement de révolte de 1963 qui eut pour conséquence l'exil de Khomeyni en Irak. Le régime du chah avait réussi à supprimer toute opposition démocratique, les formes extrêmes seules étant désormais susceptibles de se mobiliser au nom d'une idéologie de substitution au nationalisme qu'avait discrédité le régime impérial.

A la veille de la révolution, l'Iran se trouve dans une situation paradoxale : d'un côté, le pays connaît une période de croissance élevée ${ }^{13}$, une intense modernisation mentale et matérielle, une grande ouverture des nouvelles couches urbaines modernisées, surtout de classes moyennes, vers l'Occident et des formes de modernité occidentales, et d'un autre côté, la fermeture quasi totale du système politique, beaucoup plus nette que dans les années soixante, bloquant totalement l'accès des nouvelles couches modernisées à la scène politique. De cela naîtra un mouvement de contestation qui s'alimentera aussi à la crise du régime impérial (maladie du chah, avènement de Carter aux Etats-Unis et essor du thème des Droits de l'Homme, élimination de toutes les formes démocratiques de représentativité en Iran, discrédit total d'un système politique fondé sur les appareils de répression). L'essentiel est l'alliance malaisée des couches traditionnelles du bazar, de la fraction du clergé radicalisé sous Khomeyni ${ }^{14}$, des intellectuels de gauche et d'extrême gauche et de toutes ces nouvelles couches urbaines en voie de modernisation qui se sentaient spoliées de leur part de la modernité par le régime du chah ${ }^{15}$. De cette rencontre fragile, incertaine et totalement incohérente naîtra le mouvement unanimiste de la révolution iranienne où la quasi- 
totalité des couches urbaines se dressent contre le pouvoir impérial. Cette forme d'unanimisme est porteuse d'une vision floue et généreuse où se concilient mythiquement les mots d'ordre les plus contradictoires, à savoir justice, liberté, égalité et islam, sans qu'aucun travail de préparation doctrinale ait pu concilier ces principes. La nature même des groupes qui s'emparent du pouvoir au moment du renversement du régime impérial est hétéroclite: des personnalités comme Bazargan ${ }^{16}$, Premier ministre du gouvernement provisoire durant les neuf premiers mois qui suivirent le renversement du chah côtoient des membres du clergé, des représentant du bazar, les personnes désignées par Khomeyni, une partie de l'intelligentsia du temps du chah et qui revint en Iran après sa chute (comme Banisadr), quelques anciens membres du mouvement mossadéghiste, des personnages d'anciens mouvements fondamentalistes islamiques (Fédâîyané Eslâm, homologues iraniens des Frères Musulmans en Egypte), etc.

L'histoire réelle se décline hors du cercle du pouvoir légal, du reste polyarchique et éclaté (un premier pôle s'organise autour de Khomeyni, un autre, autour de Bazargan, un troisième autour de l'ayatollah Béhechti, d'autres groupes se forment dans les régions et s'imposent en vertu de leur enracinement local...). Mis à part le groupe qui soutient Bazargan, les autres, tout disparates qu'ils sont, partagent le même credo antidémocratique et la même allégeance à Khomeyni, formant le noyau dur du Hezbollah. La dénomination est d'origine coranique ("Parti de Dieu "), mais l'expression prend rapidement une connotation nettement péjorative chez tous ceux qui subissent les assauts de cet ensemble plus ou moins informe, financé par l'Etat comme un mouvement paramilitaire destiné à intimider, voire supprimer physiquement les opposants tout en instaurant au nom de l'islam un néopuritanisme religieux qui se réclame de Khomeyni et de sa prétention à islamiser radicalement la société iranienne. Ce mouvement se dote d'une réalité institutionnelle sous le nom de Parti de la République Islamique ${ }^{17}$. Il encadre des jeunes d'origine sociale inférieure, ceux issus des paysans dépaysannés, les membres des Comités (organisations d'origine locale qui ont assuré pendant la révolution la sécurité au niveau du quartier, de l'usine, de la ville... et qui ont été progressivement " épurées " et intégrées dans l'appareil répressif du nouvel Etat), bref une partie de ceux qu'on appellera par la suite les " déshérités " (mostaz'afin).

Comme toute forme de populisme, celui de Khomeyni tente de se doter d'une identité, d'abord par la manipulation de l'espérance des uns et des autres, puis par la désignation d'un ennemi commun, l'impérialisme américain, qu'on dénomme selon une terminologie d'origine coranique, l'Arrogance Mondiale (estékbâré djahâni), contre laquelle se dirige toute la hargne des uns et des autres. La guerre intervient comme facteur aggravant, mobilisant dans une indistinction lourde de sens le sentiment national et islamique dont le mélange donne naissance à une attitude effervescente, les ressources du sacré islamique nourrissant la conscience nationale et inversement, sous l'égide de Khomeyni qui cumule, en sa personne, la dimension politique (il est le promoteur de la révolution) et religieuse (il est grand-ayatollah, promu, depuis la révolution, imam, c'est-à-dire personnage suréminent doté d'une sacralité sui generis, du fait même de sa proximité avec le Douzième Imam occulté).

Bassidje

A peine après le renversement du chah et l'instauration du régime révolutionnaire, le nouveau pouvoir cherche à créer une organisation populaire où les partisans de l'islam 
défendraient la révolution; organisation qui traquerait les contre-révolutionnaires et au début, surtout les partisans du chah, et qui consoliderait l'ordre issu de la révolution. Bassidje est né de cette volonté de sauvegarder une révolution encore fragile contre les tenants de la " contre-révolution ", surtout les Tâghoutis, c'est-à-dire les partisans malfamés du régime du chah. Bassidje, littéralement " Mobilisation "18, est créée peu après le renversement du chah pour mettre la jeunesse populaire au service de la révolution. Juste après le commencement de la guerre avec l'Irak (1980) elle est intégrée au sein de l'Armée des Pasdaran, autre organisation militaire qui a vu le jour juste après la révolution pour la protéger contre les monarchistes et qui s'est constituée en force armée, indépendante du corps de l'armée traditionnelle, soupçonnée d'avoir des partisans du chah dans sa hiérarchie supérieure ${ }^{19}$. Formé de recrues volontaires, fort de quelque quatre cent mille adhérents, le Bassidji ${ }^{20}$ se fait remarquer par son dévouement total et son absence de crainte face à la mort. Il sert souvent dans la stratégie de chair à canon de l'Iran, dans des attaques à fort taux de perte en vie humaine contre une armée irakienne mieux équipée mais moins motivée que la jeunesse révolutionnaire iranienne, du moins dans les premières années de la guerre.

S'échelonnant sur plus de huit ans (1980-1988), la guerre remet en cause, sur de nombreux plans, le déroulement même de la révolution et surtout, dans sa phase initiale, l'évolution même du mouvement révolutionnaire. Déjà extrêmement faible, le mouvement démocratique coiffé par Le Mouvement pour la Liberté (Nehzaté âzâdi) de Bazargan (Premier ministre dans les neuf premiers mois de la révolution) ne résiste pas à la guerre qui entraîne des réflexes unitaires de la part de nombreuses franges de la société iranienne, a priori peu favorables au Hezbollah. Au nom de la défense de la patrie musulmane et de l'islam révolutionnaire, le groupe khomeyniste (connu sous le nom du Hezbollah, expression qui prendra de plus en plus une connotation péjorative), peut mieux accaparer le pouvoir, instrumentalisant la menace d'invasion du pays par l'Irak pour mettre fin aux fragiles revendications à l'autodétermination d'une société fraîchement dégagée de la dictature impériale. C'est sur ce fond de menace d'invasion, au sein d'une révolution elle-même sur le qui-vive, que voit le jour l'acteur bassidji, fer de lance du pouvoir révolutionnaire, non seulement dans la guerre contre l'Irak mais aussi dans le jugulement de l'opposition au sein même de la société iranienne; et ceci de double manière: d'une part, par l'intervention directe des jeunes Bassidji dans la répression physique des opposants, d'autre part, par la légitimation du pouvoir khomeyniste où des jeunes prêts à sacrifier leur vie pour la préservation du régime apportent la preuve éclatante de la " légitimité " du pouvoir hezbollah, à une société sans capacité de se défendre, épuisée par sa mobilisation pendant la révolution et ne trouvant d'autre solution que le retrait dans la vie privée ${ }^{21}$.

<!--SPIP--> Le modèle bassidji

Dans ce contexte d'une révolution menacée de toutes parts, d'un côté par la guerre, par la contre-révolution à l'intérieur du pays, que ce soit par les tenants de la droite favorable au chah, ou par les partisans de l'extrême gauche, apparait un type de personnage jeune (une vingtaine d'années, quelquefois à peine plus de 18 ans), voire très jeune (à partir de 14, voire 12 ans), qui est marqué, aux yeux d'une grande partie de la révolution iranienne, et surtout, des media occidentaux, par une démesure spécifique : son aptitude à se sacrifier au service de la révolution, ceux qu'on a appelé " 
les fous de Dieu " et qui identifient, en la personne de Khomeyni, le saint, le savant et le dirigeant politique par excellence.

Le jeune qui se déclare prêt au sacrifice est pris en charge par Bassidje, il forme le plus gros des bataillons de cette organisation qui verra, au plus fort de la révolution, le nombre de ses adhérents atteindra le chiffre de près d'un demi-million ${ }^{22}$.

Trois types d'acteurs existent au sein de Bassidje. En premier lieu, ceux qui sont habités par un désespoir profond du fait de l'état dans laquelle se trouve la révolution. Celle-ci incarne à leurs yeux le sacré islamique sensu eminenti et la menace qui se dirige sur elle; le sentiment qu'il leur sera malaisé, voire impossible de redresser les choses, entraîne une sombre vision mortifère de leur part. La mort devient de plus en plus envahissante, à mesure que la révolution s'enlise et que le marasme économique doublé de l'échec de la révolution dans la construction d'une nouvelle société devient une évidence irrécusable aux yeux d'un nombre grandissant de personnes. Ce désarroi rend compte de l'adhésion massive d'une partie des jeunes révolutionnaires à une religiosité mortifère au sein de Bassidje, où ils cherchent leur salut en martyrs. Ce groupe d'acteurs désespérés, on le qualifiera de martyropathe ${ }^{23}$. La violence qui l'habite et qui est une conséquence de son profond désespoir, a une dimension fondamentalement expressive. Les martyropathes forment vraisemblablement dix à quinze pour cent des adhérents de Bassidje.

A côté de l'acteur martyropathe, il en existe deux autres. D'une part, les adolescents qui se font enrôler dans Bassidje et l'armée des Pasdaran pour affirmer leur autonomie visà-vis des parents et devenir adultes : chez eux la guerre est comme un rite de passage, de l'adolescence à l'âge adulte, qui opère de manière précoce. La dimension ludique de la guerre intervient dans leur adhésion à Bassidje, et surtout la manipulation de leur affect religieux et leur identité sociale par les organisations affiliées à Bassidje au sein de l'école, pendant la guerre, y joue un rôle tout aussi important. Chez les jeunes ludiques, l'aspect expressif est fondamental dans leur attitude, à cette différence près, par rapport aux martyropathes, que c'est dans la vie et non pas dans la mort qu'ils entendent se réaliser. D'autre part, Bassidje joue un rôle important comme tremplin social pour tous ceux qui prennent part à la guerre au sein des organisations révolutionnaires afin de bénéficier d'une promotion socio-économique rapide, dans une société en voie d'appauvrissement où l'ascension sociale se révèle de plus en plus difficile par les voies normales ${ }^{24}$. Bassidje et l'Armée des Pasdaran facilitent grandement aux jeunes l'accès à des postes de commandement dans cette armée mais aussi dans d'autres organismes d'Etat ou dans le secteur public qui s'est largement étendu suite à la nationalisation des biens des Taghoutis (la classe dirigeante du temps du chah qui s'est expatriée pendant la révolution). Ce groupe des "opportunistes " forme la grande majorité des jeunes adhérents de Bassidje. Leur attitude est dictée par des motifs non pas expressifs, mais instrumentaux. Ils optent pour Khomeyni et sa version de l'islam moins selon une logique de conviction, qu'à partir du constat qu'il est le plus fort, qu'il peut leur apporter la promotion sociale et leur faciliter l'accès aux élites.

Bassidje commence par être une organisation bâclée, créée pour la sauvegarde de la révolution dans les premières semaines après le renversement du régime impérial, pour devenir progressivement, avec la guerre, une institution de grande envergure, intégrée dans l'Armée des Pasdaran, mais dotée de son autonomie relative pour ce qui est de son mode de recrutement (fondé sur l'adhésion volontaire des jeunes) et pour 
son idéologie légitimante (ce n'est pas l'obligation légale de faire son service militaire, mais la volonté " morale " de se dévouer à la révolution islamique qui doit motiver ses adhérents).

Financièrement, Bassidje est prise en charge par l'Armée des Pasdaran, mais elle bénéficie des " dons " (souvent imposés aux divers organismes étatiques ou privés par le pouvoir) et des mannes financières d'organismes para-étatiques tels que la Fondation des martyrs ${ }^{25}$. D'autres institutions para-étatiques, aux finances soustraites au contrôle de la Cour des comptes iranienne pendant toute la guerre, apportent leur contribution à la guerre, au service de Bassidje et de l'Armée des Pasdaran ${ }^{26}$.

Bassidje présente une spécificité dans cette société post-révolutionnaire où les rêves utopiques d'une modernité morale et indolore se mêlent inextricablement au vertige de fin du monde pour une partie de la jeunesse qui ne sait pas concilier son utopie du début de la révolution avec la réalité de plus en plus inclémente et dégradante de la post-révolution. En s'enfermant dans Bassidje, cette jeunesse occulte la disjonction entre ses rêves révolutionnaires et la crasse réalité post-révolutionnaire où la guerre aggrave encore plus l'évolution régressive de l'économie. Autant dire qu'une logique de désespoir spécifique nourrit, dans les premières années de la guerre, cette jeunesse éprise du rêve révolutionnaire et secouée par le devenir effectif de la révolution. Un gouffre le sépare de l'avenir rêvé pendant la révolution.

$\mathrm{Au}$ sein de cette organisation, soutenue et financée par l'Etat, la composition selon la classe d'âge est disparate : $y$ adhèrent en grande majorité des jeunes, mais aussi des adolescents, et des gens du troisième âge. De même, les motivations ne sont pas les mêmes. L'origine sociale des adhérents est surtout celle des classes inférieures (et quelquefois moyennes-inférieures) urbaines, la jeunesse scolarisée formant la majorité de ses recrues. Pourtant, ce qui décide de l'appartenance des jeunes à un groupe ou à un autre au sein du Bassidje, est moins l'origine sociale que le sentiment que l'on nourrit à l'égard de la révolution : quelquefois, des jeunes de la même famille adhèrent à des groupes différents, voire même antagoniques ${ }^{27}$. Ce qui semble un facteur important, est l'attitude individuelle devant la révolution et son devenir. La jeunesse rurale adhère pas à Bassidje, moins par conviction idéologique que par souci de s'ouvrir une voie vers le monde urbain ou par désir de "s'éclater ", de vivre intensément et dans un monde d'abondance sa jeunesse. L'appartenance aux couches urbaines est la condition sine qua non pour faire partie de ceux qui adhèrent à Bassidje pour des motifs idéologiques. En effet, pour être un " révolutionnaire désespéré " (martyropathe dans notre terminologie ; cf. Infra), il faut avoir été préalablement modernisé et déstructuré. Le jeune révolutionnaire ne peut se culpabiliser que s'il a le sentiment d'être un ego constitué, ce qui signifie la désarticulation de la charpente communautaire, laquelle désarticulation s'est faite principalement dans les grandes zones urbaines avant la révolution.

$\mathrm{Au}$ sein de Bassidje, on assiste à une manipulation systématique des jeunes par l'Etat islamiste. Au préalable, tout est fait pour encourager les jeunes à y adhérer. Le pouvoir n'y envoie pas les forces humaines uniquement pour nourrir Bassidje en chair à canon, mais aussi pour trouver un principe de légitimation, arguant du fait qu'un pouvoir pour qui les jeunes sont prêts à mourir ne saurait être illégitime. En réalité, les martyropathes meurent en désespoir de la non-réalisation de l'utopie révolutionnaire et pour eux, il s'agit d'une affaire existentielle. Mais le pouvoir donne un sens politique à ce désespoir et en sort revigoré par sa manipulation. Il instrumentalise ce désespoir 
pour se consolider, face à une société que traumatise et intimide l'engagement extrémiste de ses jeunes. Le désespoir des uns est ainsi manipulé pour la répression des autres.

Bassidje est le creuset où s'effectue, pendant quelques années, la confusion entre le registre de la société et de l'Etat. Ceci aurait été inconcevable dans la communauté chiite traditionnelle où ce qui est du ressort de l'Etat était identifié à une logique de violence et de peur et ce qui relève du sacré communautaire était perçu selon une logique du Nous par rapport au Eux étatique ${ }^{28}$. Par contre, l'adhésion à Bassidje est volontaire dans la grande majorité des cas. De même, chez le groupe martyropathe, la volonté de mourir est un fait d'évidence qu'exploite Bassidje, mais qu'elle n'invente pas. La mise en œuvre de la martyropathie se fait en complicité entre l'Etat et le groupe volontaire des martyropathes qui y trouvent l'expression de leur aspiration mortifère. Si la " confusion " entre le bas (la société) et le haut (l'Etat) est désormais possible, c'est parce que la structure communautaire s'est déjà largement désarticulée et qu'à sa place émerge progressivement un être en voie d'atomisation ${ }^{29}$. On est dans une formation sociale, en transition vers une nouvelle forme d'organisation sociale où le volontariat des jeunes dans Bassidje est le signe de l'émergence d'un " quasi-individu "30 dans cette société. En même temps, Bassidje exprime le désarroi d'une jeunesse qui ne sait plus lire ce qui est du registre de l'Etat ou de la société et qui, dans cette confusion mentale, mélange, sous l'effet de l'effervescence révolutionnaire du début qu'entretient artificiellement Bassidje en son sein, les domaines respectifs du pouvoir et de la société. La confusion est due, en particulier, à la déstructuration de l'ancienne formation sociale et à l'émergence chaotique de la nouvelle pendant une révolution qui a dangereusement accrue l'emprise de l'utopie sur le réel. La dynamique de Bassidje se nourrit, du moins dans les premières années, de la source vive de cette société révolutionnaire en pleine ébullition. Son mode d'organisation relève de l'Etat. Mais les martyropathes ne perçoivent pas Bassidje comme une organisation étatique. Ils élisent cette organisation comme une nouvelle " famille ", ils " se marient " avec Bassidje ; en son sein, ils se sentent frères (barâdar) avec les autres martyropathes en quête de mort. Ils la voient comme l'ombre portée de Khomeyni, sanctifiée par sa bénédiction, le lieu saint où se produit la jonction avec l'Imam du temps. Khomeyni rend ainsi possible la manipulation des Bassidji par le nouvel ordre répressif.

Pour les jeunes ludiques, Bassidje est une famille d'élection. Ils rompent avec leur famille naturelle et la rejoignent pour y trouver une nouvelle identité, volontairement construite, dans l'affrontement avec la mort. En cette période de guerre où les possibilités d'une ascension autonome sont bloquées, le jeune qui arrive sur le marché du travail n'a d'autre lieu de promotion que Bassidje. Avide de promotion sociale, il trouve en son sein l'atmosphère propice à son affirmation de soi par ses prouesses guerrières, et surtout, maintes occasions pour vivre intensément.

Pour être efficace, Bassidje se dote d'une organisation qui entend quadriller la société et inciter les jeunes à y adhérer. Parmi les instruments puissants de cet encadrement, se trouvent les Associations islamiques. Celles-ci naissent pendant la révolution. Ce sont des groupements de quartier ou de localité qui s'assignent pour tâche concrète de gérer, au quotidien, les affaires courantes (prévention du vol, fonction de police locale, etc.). Après la révolution, ces associations sont progressivement noyautées par l'Etat et par ses divers organismes répressifs ${ }^{31}$. Elles jouent alors un rôle essentiel dans 
l'épuration des éléments " contre-révolutionnaires " de l'administration, et contribuent au recrutement des volontaires pour Bassidje.

L'un des lieux de recrutement de ces " associations ", ce sont les établissements scolaires où elles s'établissent au sein d'une hiérarchie autonome (elles dépendent d'une section spéciale du Ministère de l'éducation nationale) et où elles gagnent l'adhésion de certains lycéens qui deviennent, de facto, des interlocuteurs du pouvoir, s'assurant, par cette participation, des succès scolaires qui sont indépendants de leurs résultats aux examens.

Par ailleurs, la propagande idéologique joue un rôle moteur au sein de Bassidje. Un clergé " made in Bassidje " est préposé à cette tâche qui joue sur le sentiment d'invulnérabilité des adolescents et sur leur volonté d'héroïsme.

L'une des tâches où excelle Bassidje, consiste à créer un sentiment d'appartenance des jeunes à la révolution contre le reste de la société. On y développe la vision de soi de la " caste des Purs " contre une société de plus en plus impure: dans la réalité quotidienne, les gens se distancient des slogans révolutionnaires dont l'inanité se révèle à mesure que la situation économique s'aggrave et que l'on perd l'espérance dans un avenir lumineux. Quant à Bassidje, elle donne aux jeunes le sentiment de préserver l'utopie révolutionnaire, cette fois contre une société de plus en plus incrédule.

Dans Bassidje les adolescents (de douze à seize, dix sept ans) se voient accorder un statut de quasi-adulte, voire même, de " sur-adulte " : on les glorifie de s'être haussés, par leur engagement volontaire, au-dessus des adultes, des parents qui refusent, eux, de s'enrôler ${ }^{32}$.

Les adolescents ne portent pas d'uniforme différent des autres Bassidji ; ils deviennent les égaux des adultes et les surpassent quelquefois en prestige. Séparation et hétérogénéité se dissolvent, au sein de Bassidje, dans le statut unitaire conféré aux adolescents et aux adultes, aux jeunes et aux vieux, aux pratiquants de stricte obédience et à ceux qui pratiquent de manière plus désinvolte.

Bassidje encadre d'une autre manière encore, celle-là positive, les jeunes nonmartyropathes qui la rejoignent. Il rémunère ces chômeurs, leur procure la possibilité d'exercer leur créativité au péril de leur vie. Mille subterfuges, des trouvailles sans nombre jalonnent les combats où l'ingéniosité des jeunes Bassidji trouve un immense champ d'application. On se " réalise " dans le maniement des mines. Ce n'est pas le sentiment mortifère qui motive l'adhésion de ce groupe de jeunes à Bassidje, mais le besoin de travailler, et de se valoriser dans le travail.

A mesure que s'use l'ardeur des martyrs, des mécanismes compensatoires, à base d'incitation matérielle et de coercition, sont mis en place par l'Etat, pour canaliser les jeunes vers le front. Ceux qui y sont restés quelque temps, ont un accès rapide aux postes de " renseignements " dans l'administration. Ils remplissent un rôle similaire à celui de la Savak sous le chah. Les Bassidji qui veulent faire des études universitaires, ainsi que les jeunes membres de leur famille, bénéficient d'un quota à l'université. L'accès aux coopératives des martyrs où les prix pratiqués sont très avantageux par rapport à ceux du marché, est réservé aux Bassidji blessés, à leur famille et à celle des martyrs bassidji qui bénéficient d'autres avantages matériels ${ }^{33}$.

Quoiqu'il en soit, le bassidji qui va sur le front et fait la guerre contre l'armée irakienne est un homme, jeune ou vieux, mais homme. Il existe, certes, des groupes de jeunes 
femmes bassidji (les " sœurs " bassidji), mais leur rôle se cantonne soit dans les soins médicaux, soit dans l'apport de l'aide aux jeunes qui participent directement aux combats, soit enfin dans la répression des femmes " mal-voilées " dans les zones urbaines ou encore, dans l'intimidation des parents des martyrs qui pensent que leurs jeunes sont morts injustement sur le front du fait de leur manipulation par le pouvoir islamiste (pendant le cérémonies de deuil, sous couvert de célébrer le souvenir des martyrs, ces " sœurs " étouffent en réalité les manifestations de protestation ou de mauvaise humeur des parents ou des connaissances). La femme bassidji ne prend pas directement part à la guerre. Cela est dû non pas à l'absence de volonté, de la part de certaines jeunes filles, de prendre le fusil, mais aux interdictions du pouvoir islamiste qui entend ne pas contribuer à l'égalisation des conditions entre hommes et femmes. Les femmes martyres sont celles qui le sont devenues du fait de leur mort sous les bombardements irakiens ou dans l'exercice de leurs fonction de soignante à l'hôpital ou pour toute autre raison liée à la guerre.

\section{NOTES}

1. Les statistiques sont à prendre avec prudence. On estime à quelque 500000 morts et blessés les pertes iraniennes, dont 125000 pour l'armée, le reste provenant de l'Armée des Pasdaran et des volontaires de Bassidje, et à plus d'une centaine de milliers, les invalides de guerre. Cf. Mehdi Amani, Les effets démographiques de la guerre Iran-Irak sur la population iranienne, Institut National d'Etudes démographiques, Paris, 1992.

2. Ces faits sont longuement développés dans Farhad Khosrokhavar : Le rêve impossible, une approche anthropologique de la révolution iranienne, Paris, L'Harmattan, 1996 ; L'islamisme et la mort, le martyre révolutionnaire en Iran, Paris, L'Harmattan, 1995 ; L'utopie sacrifiée, sociologie de la révolution iranienne, Paris, Presses de la FNSP, 1993. Cf. Aussi Jean-Pierre Digard, Bernard Hourcade, Yann Richard, L'Iran au XXe siècle, Paris, Fayard, 1996.

3. D'autres personnes comme Tâléghâni, Khomeyni, Banisadr, Motahhari, etc., jouent un rôle important dans d'autres couches de la population, mais chez la jeunesse urbaine, le rôle de Shariati est incomparablement plus grand. Pour un recueil de leurs textes cf. Mahmud Taleqani, Mortéza Mutahhari \& Ali Shari'ati, Jihad and Shahâdat. Struggle and Martyrdom in Islam, Texas, Institute for Research and Islamic Studies, 1986.

4. Cf. Mary Hegland, " Two images of Husain : Accomodation and Revolution in an Iranian village ", in Religion and Politics in Iran, N. R. Keddie (ed.), London, 1983 ; Le Discours populaire de la révolution iranienne, op. cit.

5. Cf. Ali Shari'ati (Shariati), Histoire et destinée, textes choisis, Paris, Sindbad, 1982. 6. Cf. infra.

7. Cette section n'a aucune intention exhaustive. Elle tente simplement de situer la place du martyre au sein de la société iranienne et les raisons qui ont abouti à l'emprise de l'islamisme khomeyniste sur la société iranienne qui avait subi une modernisation intense et despotique sous le régime du chah. 
8. Sur 10 musulmans dans le monde, grosso modo 9 sont sunnites, appartenant à l'une des quatre écoles officielles du sunnisme. Les chiites sont de loin minoritaires et se répartissent en plusieurs groupes dont les principaux sont les chiites duodécimains (à douze imams) formant l'écrasante majorité des chiites iraniens, et les Ismaéliens. Cf. Mohammad R. Djalili, Religion et révolution, Paris, Economica, 1981 ; Moojan Momen, An introduction to Shi'i Islam, New Haven, Yale University Press, 1985 ; Yann Richard, L'islam chi'ite, Paris, Fayard, 1991.

9. En particulier les écrits d'Hamid Algar, Religion and State in Iran, 1785-1906, Berkeley, University of California Press, 1969 ; The Islamic Revolution in Iran, Londres, Open Press, Muslim Institute, 1980, qui donnent une vision tendancieuse du chiisme iranien.

10. Hormis l'intermède de Nader Chah, au XVIIIe siècle, qui voulait ramener l'Iran au sunnisme, au besoin en décrétant le chiisme comme la cinquième école sunnite, ce qui occasionna le départ des ulémas chiites vers l'Irak.

11. Sur les relations entre la " hiérocratie " chiite et le pouvoir politique jusqu'à la fin du XIXe siècle, voir Saïd Amir Arjomand, The Shadow of God and the Hidden Imam : Religion, Political Order and Societal Change in Shi'ite Iran from the Beginning to 1890, Chicago, The University of Chicago Press, 1984 ; (sous la direction de), Authority and Political Culture in Shi'ism, Albany, Suny Press, 1988 ; pour la période pahlavi, voir Chahroukh Akhavi, Religion and Politics in Contemporary Iran, Albany, Suny Press, 1980.

12. Certes, de nombreux groupes de classes moyennes, formés d'intellectuels (comme l'Association des écrivains iraniens, kânouné névisandégâné irân), de juristes (comme l'Association des avocats, kânouné vokalâ), ou des organisations autonomes comme la Ligue des droits de l'homme (jam'iyaté hoghoughé bachar) ou l'Organisation nationale des professeurs d'université (sâzémâné mellié dâneshgâhiâné irân) ont joué un rôle important dans la mobilisation contre le despotisme impérial. Ces actions ont culminé en octobre 1977, dans les Dix Nuits de Poésie, à l'institut Gœthe de Téhéran, où intellectuels et poètes ont dénoncé la répression et le régime impérial. Mais, ces groupes étaient au mieux minoritaires dans le processus révolutionnaires qu'ils allaient contribuer à déclencher, et leur fragilité et le peu de soutien dont ils disposaient dans la société se révéla avec les premières mobilisations de rue contre le régime du Chah. Cf. Pour le rôle des intellectuels au début de la révolution Nasser Pakdaman : Dah shabé shé'r, bar rési va arzyâbié yék tadjrobé [Dix Nuits de Poésie : analyse et évaluation d'une expérience] 26 Février 1988, Paris.

13. Cf. Homa Katouzian, Political Economy of Modern Iran, 1926-1979, New York, New York University Press, 1980 ; Robert E. Looney, Economic Origins of the Iranian Revolution, New York, Pergamon Press, 1982 ; Mohammad H. Malek, The Political Economy of Iran under the Shah, London, Croom Helms, 1986.

14. Sur cette dimension de l'alliance entre ces couches plus ou moins traditionnelles de la société iranienne, voir Ahmad Ashraf, "Bazar-Mosque alliance : the social basis of revolts and revolutions " in International Journal of Politics, Culture and Society, 1 (4), 1988.

15. Sur cette dimension du mouvement révolutionnaire, voir Paul Vieille, Farhad Khosrokhavar, Le discours populaire de la révolution iranienne, Paris, Contemporanéité, 1990 (deux tomes).

16. Cf. Mehdi Bazargan (en persan), Enghélâbé irân da do harékat [La révolution iranienne en deux mouvements] où il analyse le renversement de la situation peu après 
la révolution, surtout la mainmise du Hezbollah et de Khomeyni sur l'Etat postrévolutionnaire. Cf. Aussi Abolhassan Banisadr, L'espérance trahie, Paris, Papyrus, 1982.

17. Pour tous ces problèmes, voir Shaul Bakhash, The Reign of the Ayatollahs. Iran and the Islamic Revolution, Londres, Tauris, 1985 ; Ali Rahnema, Farhad Nomani, The Secular Miracle, Londres, Zed Books, 1990 ; L'Utopie Sacrifiée, Sociologie de la révolution iranienne, op. cit.

18. L'expression complète était " Bassidjé ârtéché bist meliouni " [mobilisation pour une armée de vingt millions (de membres)], c'est-à-dire la quasi-totalité des jeunes iraniens susceptibles d'être appelés à la défense du pouvoir révolutionnaire.

19. Pendant la guerre contre l'Irak, il y a eu deux corps d'armée, l'armée de la République Islamique d'Iran et l'armée des Pasdaran au sein de laquelle Bassidje a préservé sa spécificité.

20. Cf. L'islamisme et la mort, le martyre révolutionnaire en Iran, op.cit., Paul Vieille : " L'institution chiite, la religiosité populaire, le martyre et la révolution " in Peuples Méditerranéens, 16, 1981 ; H.G. Kippenberg, "Jeder Tag 'Ashura, jedes Grab Kerbala : Zur Ritualisierung der Strassenkämpfe im Iran " in Kurt Greussig (dir.), Religion und Politik im Iran, Frankfurt am Main, 1981.

21. Les organisations d'extrême-gauche (les Modjâhédine et, dans une moindre mesure, l'extrême-gauche laïque et marxiste) sont marginalisées après la rupture frontale avec le régime en juin 1981 ; quant aux royalistes, leur sort est scellé dans les premiers mois de la révolution, avec l'épuration de l'armée et la fuite de leurs leaders à l'étranger, avant même la révolution. Les organisations politiques des classes moyennes, le Nehzaté âzâdi de Bazargan, le Djebhé yé melli (le Front National des mossadeghistes), sont anémiées, la répression du chah les ayant refoulées dans une marginalité, dont elles n'ont pu sortir pendant la révolution.

22. Bassidje compte quelque quatre cent mille adhérents, l'armée des Pasdaran autant, l'armée traditionnelle, presque autant. L'ensemble forme le corps de bataille de la République Islamique dans la guerre contre l'Irak.

23. Cf. Farhad Khosrokhavar, " Le Pur et l'Impur " in Peuples Méditerranéens, $n^{\circ}$ 50, 1990 ; " Chiisme mortifère : les nouveaux combattants de la foi " in L'homme et la société, n 107-108, 1993 ; " Le martyre révolutionnaire en Iran " in Social Compass, vol. 43, $\mathrm{n}^{\circ} 1$, mars 1996.

24. Ils sont rétribués de cinq à huit mille toumans. Dans une économie à plusieurs taux de change, il est impossible de chiffrer avec exactitude l'équivalent en francs de ce montant. Pour fixer les idées, on peut dire que cela correspond au traitement mensuel d'un fonctionnaire moyen pendant la guerre.

25. La Fondation des Martyrs est l'un des piliers financiers de Bassidje et de l'ensemble des organisations qui financent la guerre. Les moyens financiers dont elle dispose en font un colosse économique. Elle dispose de 55 unités, dont cinq dans les industries, 11 dans l'élevage et l'agriculture, ainsi que 14 sociétés de construction de bâtiments et de routes et 26 sociétés financières. Depuis sa création, la branche économique a mis à la disposition de la Fondation 12 milliards de rials (un peu plus d'un milliard de francs au taux officiel). Dans la branche financière, 12 milliards de rials de de pièces produits et médicaux, de chirurgie dentaire, de laboratoires, de chauffage, et des pièces détachées de camion Mack et des voitures japonaises ont été distribuées : Ettélâât du 2012.1365 (1987). 
26. Cf. Bernard Hourcade, " Vaqf et Modernité en Iran : L'agro-business de l'Âstân-e qods de Mashad " in Yann Richard (dir.), Entre l'Iran et l'Occident, Paris, Editions de la Maison des Sciences de l'Homme, 1989.

27. Dans une famille populaire (cf. Thèse d'Etat, op.cit., 1992, EHESS, Paris), le fils s'est affilié au Comité et la fille, plus âgée de deux ou trois ans, aux Modjâhédine. De nombreux exemples similaires ont pu être relevés pendant la révolution.

28. Cf. Paul Vieille, Féodalité et Etat en Iran, Paris, Anthropos, 1975

29. Ceci remet en cause la thèse du traditionalisme ou du néo-traditionalisme du mouvement révolutionnaire pour la catégorie des jeunes qui y ont participé. Cf. en particulier Saïd Amir Arjomand, The Turban for the Crown. The Islamic Revolution in Iran, New York, 1988, pour qui, la révolution islamique aurait été néo-traditionnelle : elle aurait été dirigée en réaction contre la modernisation pahlavi par des acteurs traditionnels radicalisés (le clergé et les groupes associés, le bazar et d'autres couches urbaines traditionnelles) dont le mode de vie aurait été mis en danger par la modernisation.

30. Cf. Farhad Khosrokhavar, " Le quasi-individu : de la néo-communauté à la nécrocommunauté " in François Dubet, Michel Wieviorka (dir), Penser le Sujet, Autour d'Alain Touraine, Colloque de Cerisy, Paris, Fayard, 1995.

31. Pour le cas d'une ville moyenne et la transformation du Comité en organisme de répression étatique cf. Farhad Khosrokhavar : " Le Comité dans la révolution iranienne : le cas d'une ville moyenne, Hamadan " in Peuples Méditerranéens, n 9, 1979.

32. Cf. Ian Brown, Khomeini's forgotten sons, The story of Iran's boy soldiers, Grey Seal Books, London, 1990 ; Werner Schmucker : " Iranische Märtyrertestamente " in Die Welt des Islams, 27, nº 4, 1987 ; Farhad Khosrokhavar, " Bassidje, auxiliaires juvéniles de la révolution iranienne " in Cultures \& Conflits, $\mathrm{n}^{\circ}$ 18, 1995.

33. . Il en est ainsi d'appartements bon marché, de voyages en Syrie et en Libye, payés en dollars et qui valent plusieurs fois leur prix officiel sur le marché. A noter que le prix officiel du dollar est maintenu artificiellement bas pendant la guerre, mais que son accès est restreint aux officiels triés sur le volet, qui s'enrichissent ainsi rapidement. Les autres doivent s'approvisionner sur le marché libre. Vers la fin de la guerre le dollar vaut vingt fois son taux d'avant la révolution.

\section{INDEX}

Mots-clés : enfants, forces paramilitaires, kamikaze (missions suicide), conflits internationaux Index géographique : Iran

Thèmes : Bassidje, Pasdaran 